\title{
Reflexiones sobre los métodos electroquímicos de rehabilitación de las estructuras corroídas de hormigón armado
}

\author{
J.M. Miranda*, E. Otero** y J.A. González**
}

\begin{abstract}
Resumen La realcalinización electroquímica (RAE) y la extracción electroquímica de cloruros (EEC) han mostrado su capacidad de eliminar, en pocas semanas, las causas de la corrosión de las estructuras de hormigón armado (EHA), razón por la cual reciben, actualmente, una especialísima atención. La comunicación presente intenta la búsqueda de respuestas a cuestiones fundamentales que siguen planteando fuertes controversias, que se sintetizan en la siguiente pregunta: ¿Garantiza la eliminación de las causas de la corrosión el cese de la misma?. Para ello, se analizan las respuestas de electrodos limpios y precorroídos en solución que simula la fase acuosa de la red de poros del hormigón. La eficacia de los métodos de rehabilitación electroquímica depende, fuertemente, de acuerdo con los resultados obtenidos, del grado de precorrosión de las armaduras.
\end{abstract}

Palabras clave Acero. Hormigón. Rehabilitación. Realcalinización. Extracción de cloruros.

\section{Reflections on electrochemical rehabilitation methods for corroded reinforced concrete structures}

Abstract

\begin{abstract}
Electrochemical realkalinisation and electrochemical chloride removal have shown their capacity to eliminate, in just a few weeks, the causes of corrosion of reinforced concrete structures (RCS), and for this reason are currently receiving very special attention. This communication seeks to answer some fundamental questions that continue to arouse great controversy, which are summarised in the question: removing the causes of corrosion in RCS suffice to stop rusting? To this end an analysis is made of the responses of clean and precorroded electrodes in a solution that simulates the aqueous phase of the concrete pore network. According to the results obtained, the efficiency of electrochemical rehabilitation methods depends heavily on the degree of precorrosion of the rebars.
\end{abstract}

Keywords Steel. Concrete. Rehabilitation. Realkalinisation. Chloride removal.

\section{INTRODUCCIÓN}

Se tratará de recordar, no de demostrar, varias cuestiones esenciales y evidentes respecto a la durabilidad de las EHA:

- Las enormes pérdidas debidas a la corrosión ${ }^{[1]}$, una parte considerable de las cuales son atribuibles al sector de la construcción, uno de los que más influyen en el empleo y la calidad de vida.

- Entre los materiales de construcción, el hormigón, en masa y armado, supera a todos los otros materiales considerados conjuntamente.

- El estado natural del acero en el hormigón es el pasivo y, siempre que se mantenga, la corrosión es insignificante y la durabilidad de las EHA podría considerarse "casi ilimitada".

- No obstante, la carbonatación del hormigón por el $\mathrm{CO}_{2}$ atmosférico y la penetración de iones cloruros desde el ambiente hasta el nivel de las armaduras pueden destruir la pasividad de las mismas, desencadenar la corrosión y reducir drásticamente la durabilidad de las EHA.

- Las reparaciones convencionales de las EHA implican la eliminación del hormigón carbonatado o contaminado por cloruros, la limpieza de las armaduras en todo su perímetro, el reemplazamiento del hormigón afectado y, frecuentemente, la aplicación de algún método de protección que evite nuevas reparaciones ${ }^{[2]}$. El

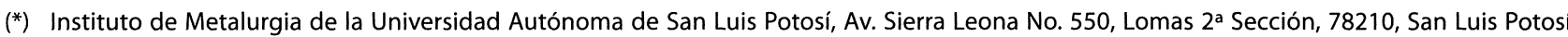
(México).

${ }^{(* *)}$ Centro Nacional de Investigaciones Metalúrgicas (CSIC), Av. Gregorio del Amo No. 8, 28040, Madrid (España). 
proceso es muy caro, y nocivo para los trabajadores y el entorno ambiental.

- Insistiendo en la idea, cabe mencionar que los costes anuales achacables en España a la corrosión en el sector de la construcción se cifran en 1.200 millones de euros ${ }^{[3]}$.

- Surgen así, como posibilidades muy sugestivas, los métodos electroquímicos de rehabilitación (MER): la protección catódica (PC), la RAE y la EEC ${ }^{[4-9]}$, aunque no sería correcto olvidar otros enfoques para prevenir los daños, como pueden ser por ejemplo, el recurso a inhibidores de la corrosión ${ }^{[3 \text { y } 10]}$ y a otros materiales conglomerantes distintos o complementarios del cemento Portland $d^{[11 \text { y } 12]}$.

El inusitado interés en los métodos electroquímicos de rehabilitación de las EHA se centra preferentemente en la RAE y la EEC, por resultar, frente a los métodos tradicionales de reparación, mucho menos costosos y más respetuosos con el entorno ambiental y la salud de los operarios; y por su carácter temporal de corta duración frente al carácter permanente y necesidad continuada de mantenimiento de la PC.

La realización práctica tiene muchas similitudes en los tres procedimientos. Se utiliza una fuente de corriente externa con un ánodo auxiliar, de la misma extensión que la estructura, inerte y formando parte de ella en el caso de la PC, externo y, frecuentemente, de escaso coste y desechable en los casos de la RAE y la EEC. Toda la red de refuerzos se conecta al terminal negativo de la fuente de corriente, forzándola a actuar como cátodo (Fig. 1). La comunicación presente, sin embargo, no va a entrar en este tipo de detalles, sino a intentar la búsqueda de respuestas a cuestiones importantes que siguen planteando fuertes controversias sobre la eficacia de la RAE y la EEC, como son las siguientes: ¿Es posible detener un proceso de corrosión ya iniciado? ¿Pueden repasivarse las EHA ya corroídas?. ¿Existe una relación entre el grado de precorrosión y la posibilidad de repasivación?. ¿Garantiza la eliminación de las causas de la corrosión el cese de la misma?, etc.

\section{MATERIALES Y PROCEDIMIENTO EXPERIMEN- TAL}

\subsection{Materiales}

Para simular las respuestas de EHA deterioradas por corrosión a la RAE o la EEC se utilizaron elec-

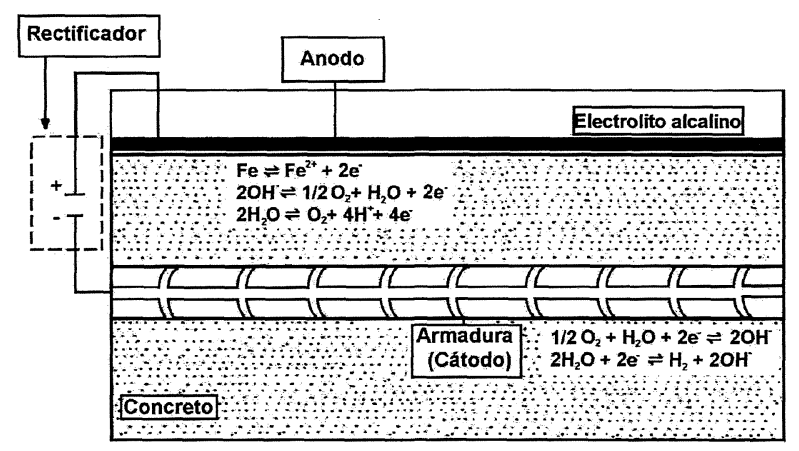

Figure 1. Esquema de las conexiones a la fuente de corriente en los métodos de rehabilitación electroquímica, incluyendo las reacciones que pueden tener lugar en el ánodo y en los refuerzos.

Figure 1. Schematic depiction of connections to the power supply in electrochemical rehabilitation methods, enclosing the reactions on the external anode and on the reinforcements.

trodos de acero limpios y con diferentes grados de precorrosión en solución saturada de $\mathrm{Ca}(\mathrm{OH})_{2}$, que simula, muy aproximadamente, la fase acuosa de la red de poros del mortero y del hormigón nuevos, es decir, sin carbonatar y sin adiciones de cloruros. Los distintos grados de precorrosión se conseguían contaminando la superficie del acero con $500 \mathrm{mg} \mathrm{de} \mathrm{Cl}^{-} / \mathrm{m}^{2}$ y manteniéndolo luego durante periodos de tiempo crecientes en cámara de humedad. Muy aproximadamente, el grado de precorrosión crecía linealmente con el tiempo de exposición.

En el curso de la investigación se realizaron también estudios paralelos en pequeñas probetas y en grandes losas de mortero de cemento, pero no se incluyen los resultados en esta comunicación.

\subsection{Técnicas de medida}

La evolución, con el tiempo, del comportamiento de los electrodos precorroídos (activos) y limpios (pasivos) se siguió a través de la medida del potencial de corrosión, $\mathrm{E}_{\mathrm{corr}}$; de la resistencia de polarización, $\mathrm{R}_{\mathrm{p}}$, para calcular la velocidad de corrosión a partir de la ecuación de Stern y Geary ${ }^{[13]}$; del trazado de las curvas de polarización, y de la respuesta a pulsos galvanostáticos de corta duración. Procedimientos todos ellos, con la excepción de la medida del $\mathrm{E}_{\text {corr }}$, que permiten precisar el estado activo o pasivo de los refuerzos. Es decir, la eficacia de los MER. Los $\mathrm{E}_{\text {corr }}$ se determinaron siempre con respecto al electrodo de calomelanos saturado. 
Reflexiones sobre los métodos electroquímicos de rehabilitación de las estructuras corroídas de hormigón armado J.M. MiRANDA, E. Otero y J.A. GONZÁlez

\section{RESULTADOS}

Las figuras $2 \mathrm{a}$ ) y $2 \mathrm{~b}$ ) muestran el aspecto de las probetas con dos grados de precorrosión muy diferentes, concretamente de 88 y $794 \mathrm{mg}$ de Fe/dm², respectivamente.

La figura 3 reproduce la evolución con el tiempo de las velocidades de corrosión ( $\mathrm{i}_{\text {corr }}$ ) para el caso de probetas limpias y con 3 grados de precorrosión diferentes, al sumergirlas en la solución saturada de $\mathrm{Ca}(\mathrm{OH})_{2}$ exenta de cloruros. Las $\mathrm{i}_{\text {corr }}$ de los electrodos precorroídos llegan a ser hasta unos 3 órdenes de magnitud mayores que las determinadas para los electrodos limpios. Las $i_{\text {corr }}$ a las que se tiende son tanto mayores cuanto más alto es el grado inicial de precorrosión.
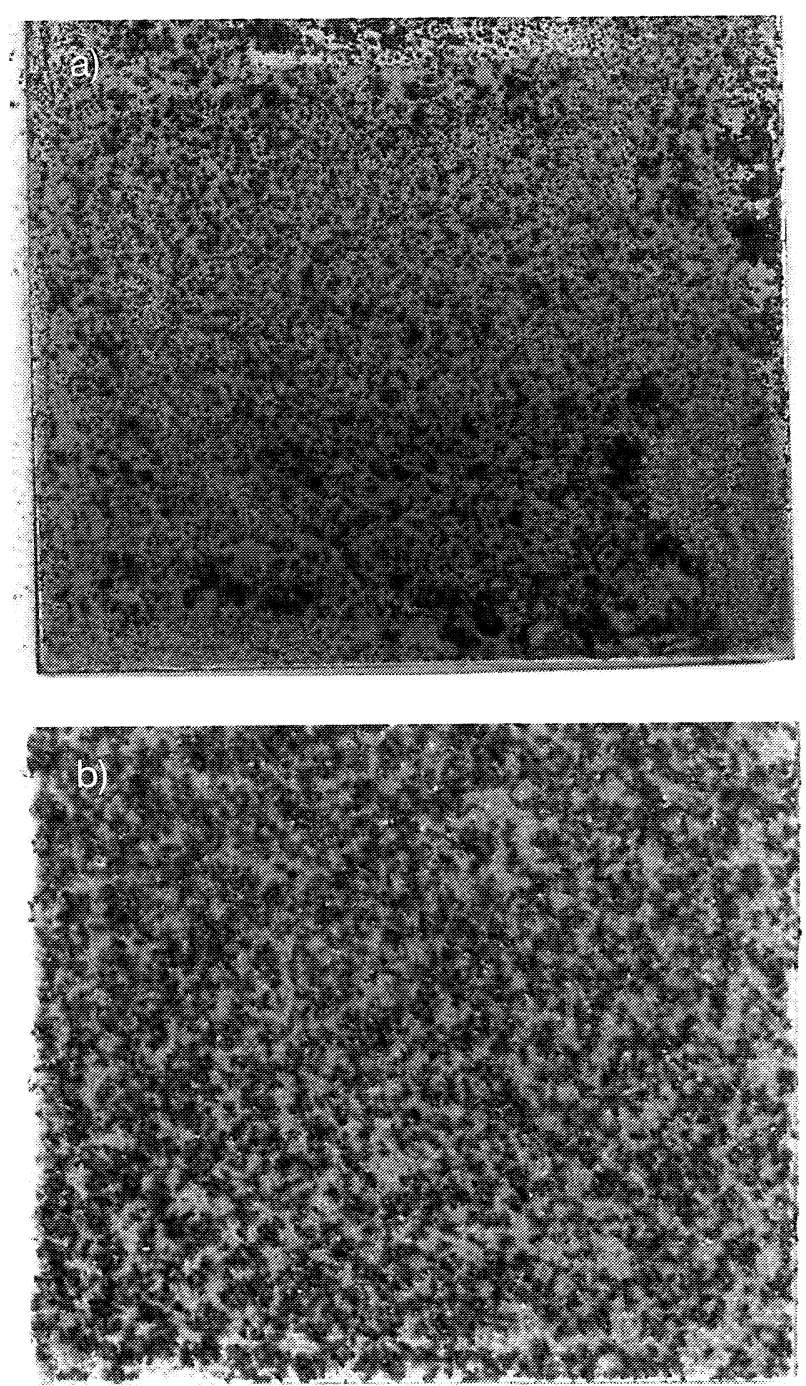

Figure 2. Aspecto de las muestras precorroídas en cámara de humedad, después de contaminadas con $500 \mathrm{mg}$ de $\mathrm{Cl}^{-} / \mathrm{m}^{2}$ : a) precorroídas un solo día; b) precorroídas $14 \mathrm{~d}$.

Figure 2. Surface aspects of steel specimens prerusted in a humidity cabinet, after contamination with $500 \mathrm{mg} \mathrm{Cl} / \mathrm{m}^{2}$ : a) prerusted 1 day; b) prerusted $14 \mathrm{~d}$.

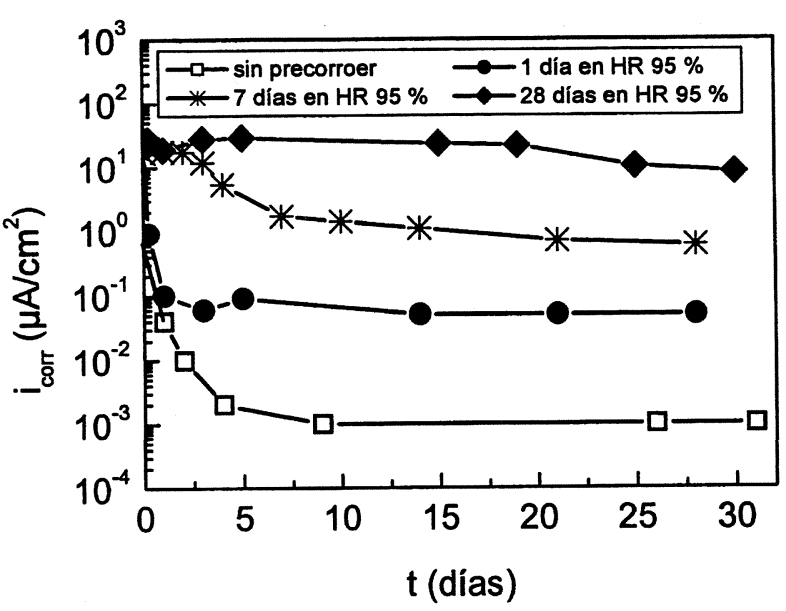

Figure 3. Evolución de las $i_{\text {corr }}$ de electrodos limpios y con distintos grados de precorrosión con el tiempo de inmersión en solución saturada de $\mathrm{Ca}(\mathrm{OH})_{2}$.

Figure 3. Evolution of $i_{\text {corr }}$ with time in a $\mathrm{Ca}(\mathrm{OH})_{2}$ saturated solution for steel electrodes clean and with different prerusting degrees.

Una apreciación más directa de la influencia del grado de precorrosión sobre la posible eficacia de los MER puede apreciarse en la figura 4, en la que se relacionan las $i_{\text {corr }}$ obtenidas tras un mes de inmersión en la solución de ensayo, con el grado de precorrosión determinado por disolución de los productos de corrosión y pesada en 3 probetas que han sufrido un tratamiento idéntico que las utilizadas en las determinaciones electroquímicas.

En la figura 5 se comparan las curvas de polarización de electrodos precorroídos y limpios al

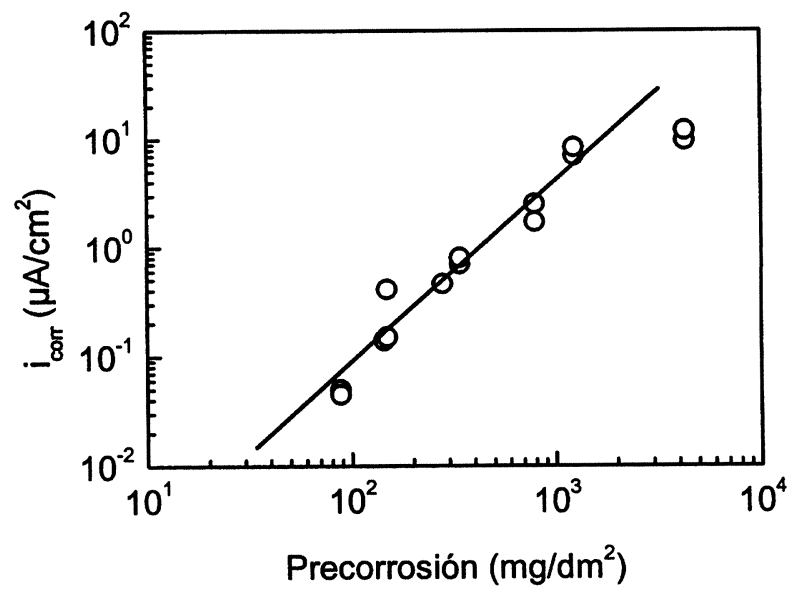

Figure 4. Relación entre el grado de precorrosión de los

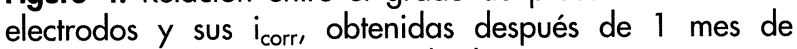
inmersión en la solución saturada de $\mathrm{Ca}(\mathrm{OH})_{2}$.

Figure 4. Relation between $i_{\text {corr }}$ values and the prerusting degrees of electrodes after 1 month of exposure in a $\mathrm{Ca}(\mathrm{OH})_{2}$ saturated solution. 


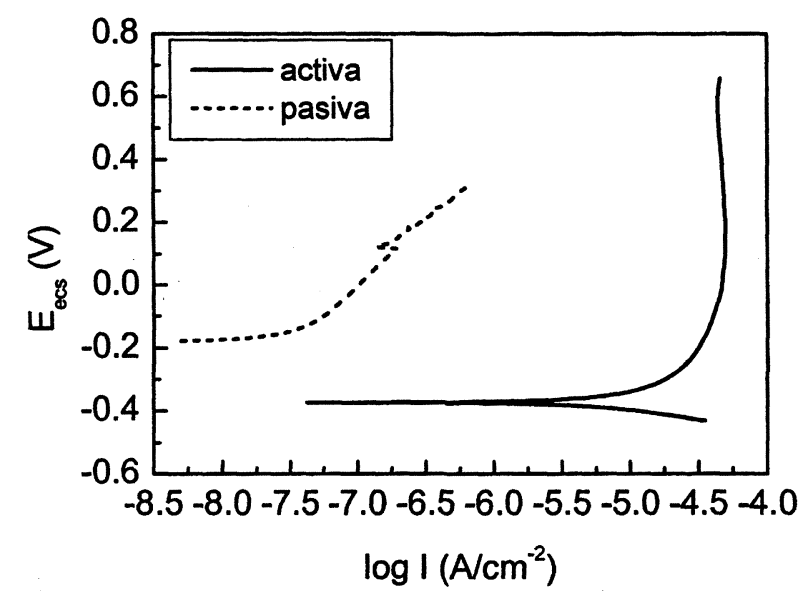

Figure 5. Curvas de polarización de electrodos limpios y precorroídos durante 4 semanas, obtenidas después de 1 mes de inmersión en la solución saturada de $\mathrm{Ca}(\mathrm{OH})_{2}$.

Figure 5. Polarization curves for the clean and 4 weeks prerusted steel electrodes obtained after 1 month of exposure in a $\mathrm{Ca}(\mathrm{OH})_{2}$ saturated solution.

término de $30 \mathrm{~d}$ de inmersión en la solución de ensayo. Puede comprobarse que las respuestas se diferencian, también, casi tres órdenes de magnitud en la rama anódica de las curvas, dependiendo del estado inicial del acero, limpio o con una capa considerable de productos de corrosión.

\section{DISCUSIÓN}

Las condiciones impuestas en los ensayos reproducen las que se conseguirían después de una rehabilitación electroquímica perfecta, pues se enfrentan electrodos con diferentes grados de precorrosión con un electrólito igual que el de la red de poros de un mortero u hormigón recién fabricado. Los diferentes grados de precorrosión (Figs. 2a) y 2b)) reproducen EHA deterioradas, en mayor o menor grado, por la corrosión, mientras que la solución de ensayo, exenta de cloruros, asegura un poder repasivador que no se podría conseguir nunca en la práctica tras la EEC, que reduce la contaminación en cloruros por debajo de los umbrales permitidos en las distintas normalizaciones, pero nunca los elimina totalmente. Quiere esto decir, que si no se consigue repasivar así el acero precorroído, tampoco la RAE ni la EEC conseguirán repasivar los refuerzos de las EHA dañadas por corrosión a las que se apliquen.

\section{1. ¿Pueden repasivarse las EHA ya corroídas?}

La observación de las figuras 3 y 5 ponen de manifiesto respuestas muy distintas de los electrodos precorroídos y limpios frente a cualquier tipo de determinación cuantitativa de la cinética de corrosión; ya se trate de las velocidades de corrosión $\left(\mathrm{i}_{\text {corr }}\right)$ calculadas a partir de medidas de la resistencia de polarización (Fig. 3), ya de la aplicación de fuertes polarizaciones como las exigidas por el trazado de las curvas de la figura 5, que muestran respuestas en corriente típicas del estado activo en las muestras precorroídas y del estado pasivo en las limpias, respectivamente. Los resultados obtenidos obligan, pues, a contestar negativamente a la pregunta que inicia este apartado.

\section{2. ¿Existe una relación entre el grado de pre- corrosión y la posibilidad de repasivación?}

Los resultados de las figuras 3 y 4 ofrecen pruebas convincentes de la gran importancia que el grado de precorrosión del acero ejerce sobre las $i_{\text {corr }}$ a las que se tiende tras una hipotética rehabilitación electroquímica. La eficacia de los MER se atenúa pues progresivamente al crecer la cuantía del deterioro inicial de la estructura, y habría que contestar afirmativamente a la cuestión planteada.

\section{3. ¿Es posible detener un proceso de corro- sión ya iniciado?}

Está demostrado que una PC correctamente aplicada puede detener un proceso ya iniciado de corrosión en las EHA, cualquiera que sea la concentración de cloruros que lo provoca ${ }^{[4}$ y 5$]$. Asímismo, reduciendo el porcentaje de saturación de los poros del hormigón hasta alcanzar resistividades $\geq 100 \mathrm{k} \Omega \mathrm{cm}$, que dan lugar a $i_{\text {corr }} \leq 0,1$ $\mu \mathrm{A} / \mathrm{cm}^{2}$, incluso en estructuras activas ${ }^{[14 \text { y } 15]}$, puede anularse prácticamente el riesgo que el proceso de corrosión supone para la durabilidad de las EHA. Los inconvenientes radican en que la PC, como ya se ha comentado, es un procedimiento permanente y, modificar la resistividad de las estructuras reales, que depende de las condiciones ambientales, no suele resultar factible.

\section{4. ¿Garantiza la eliminación de las causas de la corrosión el cese de la misma?}

Esta demostrado que el hormigón puede realcalinizarse y extraer electroquímicamente una proporción considerable de los cloruros de las EHA contaminadas por estos iones ${ }^{[6-9]} \mathrm{y}$, frecuentemente, tales resultados se presentan, ya, como éxitos. Sin embargo, el objetivo perseguido suele ser 
garantizar la durabilidad de las EHA, lo que exige eliminar los efectos de la carbonatación y los cloruros. La RAE y la EEC no pueden constituir un fin en si mismas, sino un medio para lograr la repasivación de los refuerzos. En términos clínicos, la eliminación de las causas de la enfermedad no devuelve la salud si no desaparece el daño, los efectos de las causas. Los resultados obligan a reconocer que, cuando los daños son ya considerables, la aplicación de la RAE o de la EEC no detiene la corrosión.

\section{5. ¿Qué beneficio aportan los MER?}

Volviendo a la RAE y la EEC, si los resultados evidencian su incapacidad para detener la corrosión de los refuerzos con capas considerables de productos de corrosión (Figs. 3 y 4) utilizadas como medidas preventivas, es decir, realcalinizando el hormigón antes de que el frente de carbonatación alcance el nivel de las armaduras, o extrayendo los cloruros antes de que produzcan la despasivación de las mismas, pueden evitar que se inicie el proceso de corrosión en estado activo.

\section{CONCLUSIONES}

- Existe una clara relación entre el grado de precorrosión de los refuerzos y las velocidades de corrosión de los mismos en medios que simulan la fase acuosa de la red de poros de un hormigón sin carbonatar y exento de cloruros (después de aplicados los MER).

- Ni la RAE ni la EEC son capaces de repasivar superficies de acero muy corroídas. La RAE y la EEC repasivan, en cambio, las muestras de acero con grados incipientes de corrosión.

- La RAE y la EEC no pueden considerarse como un fin en si mismas, sino como medios para lograr la repasivación de los refuerzos en EHA dañadas por corrosión. Aplicadas preventivamente, antes de que el frente de carbonatación o los cloruros alcancen el nivel de las armaduras, la RAE y la EEC pueden retrasar el inicio de la corrosión en estado activo indefinidamente, si se aplican periódicamente.
- Los MER pueden considerarse, pues, como métodos adecuados de prevención pero, estrictamente hablando, no como métodos de rehabilitación.

\section{REFERENCIAS}

[1] Report on the Committee on Corrosion and Protection (presidido por T.P. Hoar). Dept. of Trade and Industry. HMSO, Londres 1971.

[2] M. Fernández CÁnovas, Patología y Terapéutica del Hormigón Armado. $2^{a}$ Edición. Editorial Dossat, S.A. Barcelona, 1984. pp. 443-473.

[3] R. MARTínEZ, Hormigón, 38 (1998) 48-50.

[4] B.S. Wyatt y D.I. IRvine, Mater. Performance 26 (1987) 12-21.

[5] R.A. BARNHART, FHWA Memorandum, Federal Highway Administration, April 1982.

[6] J. Mietz, Publicación $N^{\circ} 24$ de la European Federation of Corrosion. The Institute of Materials, London 1998.

[7] T.K.H. Al-KadHimi, P.F.G. Banfill, S.G. Millard Y J.H. BungeY, Corrosion of Reinforcement in Concrete Construction". Eds. C.L. Page, P.B. Bamforth y J.W. Figg, Royal Society of Chemistry, Cambridge, 1996, pp. 501 . 511.

[8] A.J. VAN DEN HONDEl Y R.B. POldeR, Construction Repair, 19 (1992) 20-28.

[9] P. CASTRo, Corrosión en Estructuras de Concreto ArmadoTeoría, Inspección, Diagnóstico, Vida útil y Reparaciones, P. Castro (Ed.), IMCYT, México D.F. (1998) pp. 93-116.

[10] E. Anzola, R. Malavé, V. Barrios, D. Villarroel, A. Tiso y M. PARra, Rev. Metal. Madrid Vol. Extr. (2003) 157-161.

[11] Y. HeRnÁNDEZ, O. DE Rincón y R. FERnÁNdeZ, Rev. Metal. Madrid Vol. Extr. (2003) 137-142.

[12] R. Mejía de Gutiérrez, Rev. Metal. Madrid Vol. Extr. (2003) 250-255.

[13] M. Stern y A.L. Geary, J. Electrochem. Soc. 104 (1957) 56-63.

[14] W. López y J.A. GonzÁlez, Cement. Concrete. Res. 23 (1993) 368-376.

[15] J.A. González, J.M. Miranda y A. Cobo, Cemento Hormigón, 73 (838) (2002) 10-26. 